\title{
Prognostic performance of lymphocyte-to- monocyte ratio in diffuse large B-cell lymphoma: an updated meta-analysis of eleven reports
}

This article was published in the following Dove Press journal:

OncoTargets and Therapy

20 May 2016

Number of times this article has been viewed

Hui-Ling Sun ${ }^{1, *}$

Yu-Qin Pan ${ }^{1, *}$

Bang-Shun $\mathrm{He}^{\prime}$

Zhen-Lin $\mathrm{Nie}^{2}$

Kang Lin'

Hong-Xin Peng ${ }^{1,3}$

William C Cho ${ }^{4}$

Shu-Kui Wang'

'Central Laboratory, ${ }^{2}$ Laboratory Medicine, Nanjing First Hospital, Nanjing Medical University, ${ }^{3}$ Medical College, Southeast University, Nanjing, ${ }^{4}$ Department of Clinical Oncology, Queen Elizabeth Hospital, Hong Kong, People's Republic of China

*These authors contributed equally to this work
Correspondence: Shu-Kui Wang Central Laboratory, Nanjing First Hospital, Nanjing Medical University, 68 Changle Road, Nanjing 210006 , People's Republic of China Emailshukwang@I63.com
Purpose: The findings on the prognostic value of lymphocyte-to-monocyte ratio (LMR) in diffuse large B-cell lymphoma (DLBCL) are inconsistent. This meta-analysis was conducted to more precisely evaluate the prognostic significance of LMR in DLBCL.

Methods: This analysis combined eleven studies with 4,578 patients aiming to assess the association of LMR with overall survival (OS) and progression-free survival (PFS) in DLBCL. Data from studies directly reporting a hazard ratio (HR) with $95 \%$ corresponding confidence interval (CI) in multivariate analysis were pooled to estimate the effect.

Results: Our results suggested that patients with decreased LMR had shorter OS (HR $=1.79$, 95\% CI $=1.54-2.08, P<0.001)$ and PFS ( HR $=2.21,95 \% \mathrm{CI}=1.80-2.72, P<0.001)$ in DLBCL. Stratified analyses indicated that each confounder showed consistent prognostic value in DLBCL. There was no significant heterogeneity for PFS $\left(P_{\mathrm{H}}=0.192\right)$ and OS $\left(P_{\mathrm{H}}=0.212\right)$ among the enrolled studies.

Conclusion: This meta-analysis indicated that decreased LMR might be a marker in the prediction of poor prognosis for patients with DLBCL.

Keywords: diffuse large B-cell lymphoma, lymphocyte-to-monocyte ratio, meta-analysis, prognosis

\section{Introduction}

Diffuse large B-cell lymphoma (DLBCL) is one of the most frequent subtypes of nonHodgkin's lymphoma, accounting for $>25 \%$ of all newly diagnosed cases worldwide. ${ }^{1}$ Despite substantial advance in treatment since the introduction of rituximab, the prognosis of DLBCL remains unsatisfactory due to its aggression with heterogeneous clinical behaviors. ${ }^{2}$ A large number of studies have found multiple factors to predict the prognosis of patients with DLBCL. However, the hallmark prognostic factor is not fully confirmed in patients with DLBCL.

Systemic immune suppression is susceptible to the development of lymphoma. ${ }^{3}$ Emerging evidence has shown a close association between the host immune status and lymphoma biology, indicating that the clinical outcomes of lymphoma are associated with tumor inflammation and immunology. ${ }^{4}$ Tumor inflammation and immunology have been extensively identified to be involved in tumor biologic behaviors. ${ }^{5,6}$ Systemic inflammatory markers have also been reported to predict the survival outcomes in various solid cancers, such as C-reactive protein, neutrophil-to-lymphocyte ratio, and platelet-to-lymphocyte ratio. ${ }^{7-9}$ Considering the cost and technical limitations for clinical application, increasing studies have focused on seeking a surrogate biomarker representing the host immune status in peripheral blood that can serve as a prognostic 
factor in DLBCL. ${ }^{10,11}$ Both lymphocytes and monocytes are surrogate biomarkers of immune response and tumor microenvironment; they have been extensively identified as the prognostic factors to predict survival for DLBCL. ${ }^{12}$

Recent data have suggested that the lymphocyte-tomonocyte ratio (LMR) may predict the survival outcomes of patients with DLBCL. ${ }^{11}$ Some investigators reported that decreased LMR was linked to shorter survival in patients with DLBCL, ${ }^{13,14}$ while a few scientists suggested that decreased LMR had less association with prognosis in patients with germinal center-type DLBCL treated with rituximab, cyclophosphamide, doxorubicin, vincristine, and prednisone (R-CHOP). ${ }^{15}$ Therefore, it is essential to further illuminate the prognostic performance of LMR in patients with DLBCL. In this study, we conducted an updated meta-analysis to evaluate the impact of LMR on the prognosis of 4,587 patients with DLBCL from eleven reports.

\section{Methods}

\section{Study search}

A literature review system with the Preferred Reporting Items for Systematic Reviews and Meta-Analysis guidelines was used to search the published data. ${ }^{16}$ The literature search was carried out in the databases of PubMed and Web of Science to evaluate the association of LMR with the clinical prognosis of patients with DLBCL (updated on October 20, 2015). The following keywords are used, including "lymphocyteto-monocyte ratio", "lymphocyte monocyte ratio", "LMR", "diffuse large B cell lymphoma", "diffuse large B-cell lymphoma", "DLBCL", "prognostic", "survival", and "prognosis". Article language was restricted to English.

\section{Study selection}

Two investigators (HLS and YQP) reviewed all the candidate papers independently. Disagreements were resolved by discussion. Studies were included in the meta-analysis according to the following criteria: 1) investigated patients with DLBCL; 2) explored the association of LMR with overall survival (OS) or progression-free survival (PFS); 3 ) extracted available data of a hazard ratio (HR) with $95 \%$ CI for OS or PFS in multivariate analysis; and 4) article language restricted to English.

\section{Data extraction}

Two reviewers (HLS and YQP) reviewed each eligible study according to the inclusion criteria and extracted the available data. The extracted content included the first author's name, study country, study duration, tumor stage, cutoff value of decreased LMR, treatment method, study design, follow-up period, number of patients, and HRs with 95\% CIs for OS and PFS in multivariate analysis.

\section{Statistical analysis}

STATA software Version 11.0 (StataCorp LP, College Station, TX, USA) was used to analyze the extracted data. HRs with corresponding 95\% CIs were directly obtained from each eligible study. Both the random-effects model (DerSimoian-Laird method) and the fixed-effects model (Mantel-Haenszel method) were used to generate the pooled results. Subgroup analysis and meta-regression were performed to explore the reasons for interstudy heterogeneity. Sensitivity analysis was conducted to estimate the stability of the combined results. The publication bias of the studies was evaluated by the Egger's linear regression test. Statistical analyses were two sided, and a $P$-value $<0.05$ was considered statistically significant.

\section{Results}

\section{The selection and characteristics of the studies}

The flow chart of the study selection has been shown in Figure 1. A total of 18 studies were recorded in the initial electronic search. According to the inclusion and exclusion

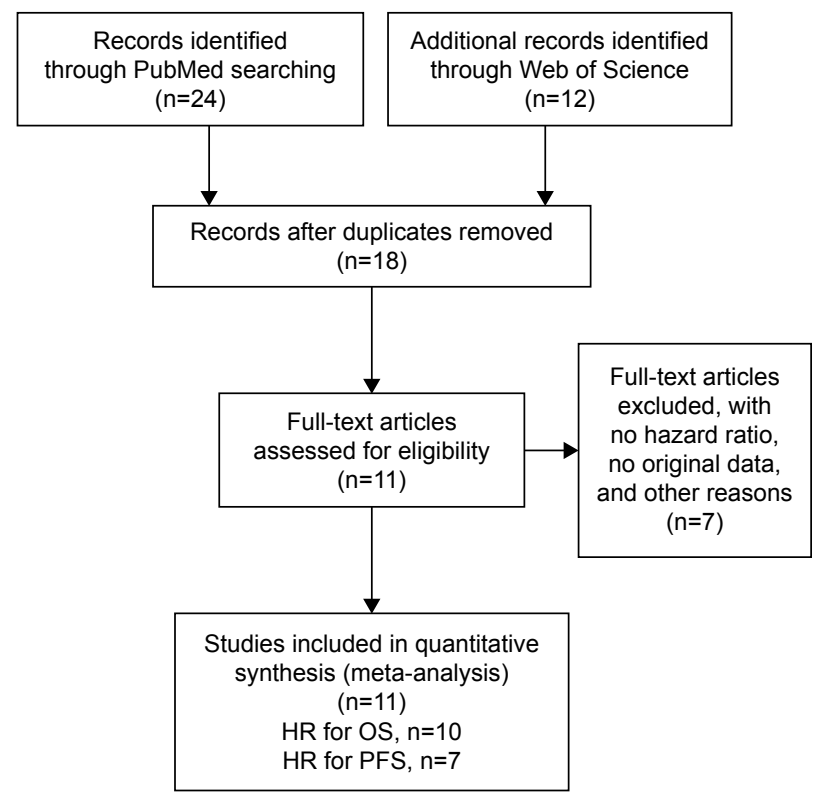

Figure I Flow chart of the eligible studies in this meta-analysis.

Abbreviations: HR, hazard ratio; OS, overall survival; PFS, progression-free survival. 
criteria, there were eleven eligible studies included in this meta-analysis. The major characteristics of these studies are shown in Table 1. These included studies with 4,578 patients were mostly published between 2012 and 2015 . There were six studies composed of two cohorts reporting the HR with $95 \%$ CI. One study included only late-stage disease (III/IV), whereas ten studies involved all the disease stages. Patients with DLBCL were treated with R-CHOP in eight studies, and patients in other studies were treated with various therapeutic approaches, including $\mathrm{CHOP}$, radiation, and surgery marked as non-R-CHOP. Ten studies with 4,441 patients with DLBCL investigated the association of LMR with OS, while seven studies with 2,100 patients explored the correlation between LMR and PFS.

\section{The association of LMR with OS in DLBCL}

Ten studies reported the association of LMR with OS in 4,441 patients with DLBCL (Table 2). The pooled results of these studies indicated that patients with decreased LMR were significantly associated with shorter OS ( $\mathrm{HR}=1.79,95 \% \mathrm{CI}=1.54-2.08, P<0.001)$, and there was no significant heterogeneity among these studies $\left(P_{\mathrm{H}}=0.212\right.$; Figure 2). Thereafter, subgroup analysis was performed according to confounders, including the country of study, cutoff value defining decreased LMR, treatment method, and sample size.

In the stratified analysis by population-based country, we found that the pooled HRs were $1.59(95 \% \mathrm{CI}=1.31-1.94)$ for patients in Western countries and $2.08(95 \% \mathrm{CI}=1.65-2.63)$ for patients in Eastern countries. Stratification by cutoff showed that decreased LMR was associated with poor prognosis for patients with both LMR cutoff $<3$ (HR $=1.65$, $95 \% \mathrm{CI}=1.38-1.98)$ and $\geq 3(\mathrm{HR}=2.12,95 \% \mathrm{CI}=1.61-2.79)$. Subgroup analysis by treatment method suggested that there were similar HRs in patients treated with R-CHOP (HR $=1.75,95 \% \mathrm{CI}=1.48-2.08)$ and non-R-CHOP ( $\mathrm{HR}=1.90,95 \% \mathrm{CI}=1.38-2.61)$. Similar results were also observed in subgroup analysis by sample size $(<400$ vs $\geq 400)$ (Table 2$)$.

\section{The association of LMR with PFS in DLBCL}

The association of LMR with PFS in 2,100 patients with DLBCL was further investigated in this meta-analysis (Table 2). A total of seven studies presented the influence of LMR on PFS in patients with DLBCL. Combined data

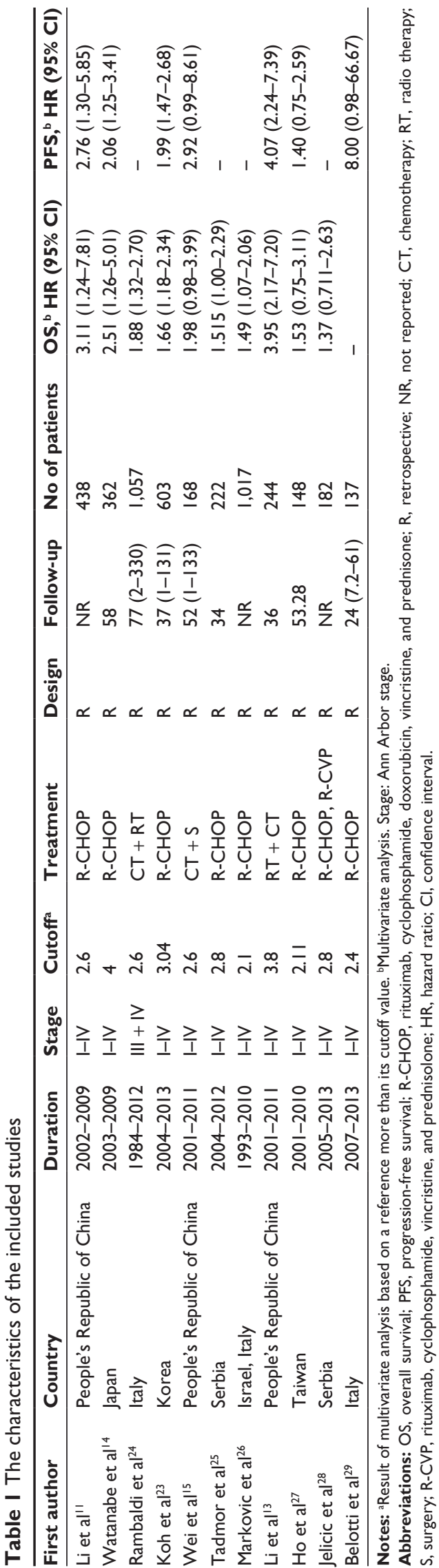


Table 2 The main results of the meta-analysis

\begin{tabular}{|c|c|c|c|c|c|c|c|c|}
\hline \multirow[t]{2}{*}{ Outcome } & \multirow[t]{2}{*}{ Variables } & \multirow[t]{2}{*}{ No of studies } & \multirow[t]{2}{*}{ No of patients } & \multicolumn{3}{|c|}{$P$-value } & \multicolumn{2}{|c|}{ Regression model, HR $(95 \% \mathrm{Cl})$} \\
\hline & & & & $P_{\mathrm{H}}$ & $P_{\mathrm{z}}$ & $\boldsymbol{P}_{\mathrm{E}}$ & Random & Fixed \\
\hline \multirow[t]{14}{*}{ OS } & All & 10 & 4,441 & 0.212 & $<0.001$ & 0.145 & $1.83(1.52-2.19)$ & $1.79(1.54-2.08)$ \\
\hline & Stratified analysis & & & & & & & \\
\hline & Country & & & 0.086 & & & & \\
\hline & Eastern & 6 & 1,963 & 0.166 & $<0.001$ & & $2.21(1.61-3.02)$ & $2.08(1.65-2.63)$ \\
\hline & Western & 4 & 2,478 & 0.740 & $<0.001$ & & $1.59(1.31-1.94)$ & $1.59(1.31-1.94)$ \\
\hline & Cutoff & & & 0.139 & & & & \\
\hline & $<3$ & 7 & 3,232 & 0.745 & $<0.001$ & & $1.65(1.38-1.98)$ & $1.65(1.38-1.98)$ \\
\hline & $\geq 3$ & 3 & 1,209 & 0.042 & $<0.001$ & & $2.44(|.4|-4.22)$ & $2.12(1.61-2.79)$ \\
\hline & Treatment & & & 0.662 & & & & \\
\hline & R-CHOP & 8 & 3,216 & 0.107 & $<0.001$ & & $1.84(1.45-2.34)$ & $1.75(1.48-2.08)$ \\
\hline & Non-CHOP & 2 & I,225 & 0.897 & $<0.001$ & & $1.90(1.38-2.61)$ & $1.90(1.38-2.61)$ \\
\hline & Sample size & & & 0.453 & & & & \\
\hline & $<400$ & 6 & 1,326 & 0.115 & $<0.001$ & & $1.97(1.4 \mathrm{I}-2.74)$ & $1.92(1.51-2.44)$ \\
\hline & $\geq 400$ & 4 & 3,115 & 0.458 & $<0.001$ & & $1.70(1.4 \mathrm{I}-2.07)$ & $1.70(\mid .4 \mathrm{I}-2.07)$ \\
\hline \multirow[t]{14}{*}{ PFS } & All & 7 & 2,100 & 0.192 & $<0.001$ & 0.226 & $2.31(1.74-3.06)$ & $2.21(1.80-2.72)$ \\
\hline & Stratified analysis & & & & & & & \\
\hline & Country & & & 0.230 & & & & \\
\hline & Eastern & 6 & 1,963 & 0.649 & $<0.001$ & & $2.25(I .7 I-2.97)$ & $2.18(1.77-2.69)$ \\
\hline & Western & 1 & 137 & - & 0.053 & & $8.00(0.97-65.98)$ & $8.00(0.97-65.98)$ \\
\hline & Cutoff & & & 0.787 & & & & \\
\hline & $<3$ & 4 & 891 & 0.254 & 0.001 & & $2.24(1.31-3.82)$ & $2.10(1.37-3.21)$ \\
\hline & $\geq 3$ & 3 & 1,209 & 0.103 & $<0.001$ & & $2.41(1.62-3.58)$ & $2.24(1.77-2.84)$ \\
\hline & Treatment & & & 0.606 & & & & \\
\hline & R-CHOP & 6 & 1,932 & 0.134 & $<0.001$ & & $2.29(1.68-3.13)$ & $2.19(1.77-2.70)$ \\
\hline & Non-CHOP & I & 168 & - & 0.052 & & $2.92(0.99-8.61)$ & $2.92(0.99-8.61)$ \\
\hline & Sample size & & & 0.534 & & & & \\
\hline & $<400$ & 5 & 1,059 & 0.104 & $<0.001$ & & $2.49(1.55-3.99)$ & $2.37(1.74-3.23)$ \\
\hline & $\geq 400$ & 2 & $|, 04|$ & 0.429 & $<0.001$ & & $2.08(1.58-2.75)$ & $2.08(1.57-2.75)$ \\
\hline
\end{tabular}

Notes: $P_{\mathrm{E}}, P$-value for Egger's test; $P_{\mathrm{H}}, P$-value for heterogeneity; $P_{\mathrm{Z}}, P$-value for $Z$ test.

Abbreviations: OS, overall survival; PFS, progression-free survival; R-CHOP, rituximab, cyclophosphamide, doxorubicin, vincristine, and prednisone; HR, hazard ratio; $\mathrm{Cl}$, confidence interval.

from these seven studies indicated that decreased LMR was obviously correlated with poor PFS (HR $=2.21,95 \%$ $\mathrm{CI}=1.80-2.72, P<0.001)$, and there was no heterogeneity among these studies $\left(I^{2}=0.0 \%, P_{\mathrm{H}}=0.192\right.$; Figure 3$)$. Subsequently, stratified analysis was also conducted on the basis

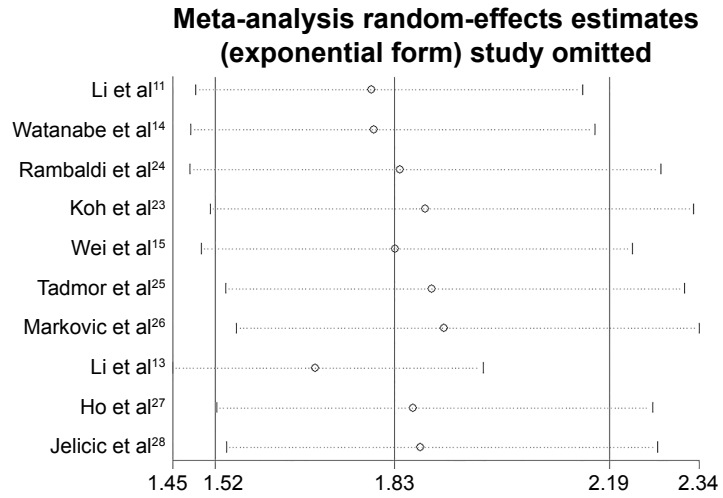

Figure 2 Forest plots of the studies assessing the HRs with corresponding $95 \% \mathrm{Cls}$ of LMR for OS.

Abbreviations: HR, hazard ratio; LMR, lymphocyte-to-monocyte ratio; OS, overall survival; $\mathrm{Cl}$, confidence interval. of the earlier confounders in PFS. Stratification showed that decreased LMR could predict poor prognosis in DLBCL regardless of the country of study (Western vs Eastern), cutoff ( $<3$ vs $\geq 3$ ), treatment (R-CHOP vs non-R-CHOP), and sample size $(<400 \mathrm{vs} \geq 400)$ (Table 2$)$.

\begin{tabular}{|c|c|c|c|}
\hline Study ID & & HR $(95 \% \mathrm{Cl})$ & $\begin{array}{l}\% \\
\text { weight }\end{array}$ \\
\hline Li et al ${ }^{11}$ & : & $2.76(1.30,5.85)$ & 7.57 \\
\hline Watanabe et al ${ }^{14}$ & 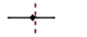 & $2.06(1.25,3.41)$ & 17.00 \\
\hline Koh et $\mathrm{al}^{23}$ & $\rightarrow$ & $1.99(1.47,2.63)$ & 47.46 \\
\hline Wei et $\mathrm{al}^{15}$ & & $2.92(0.99,8.61)$ & 3.66 \\
\hline Li et $\mathrm{al}^{13}$ & & $4.07(2.24,7.39)$ & 12.01 \\
\hline Ho et $a^{27}$ & & $1.40(0.76,2.59)$ & 11.34 \\
\hline Belotti et a ${ }^{29}$ & : & $\rightarrow 8.00(0.93,66.67)$ & 0.96 \\
\hline Overall $\left(I^{2}=31.0 \%, P=0.192\right)$ & (i) & $2.21(1.80,2.72)$ & 100 \\
\hline
\end{tabular}

Figure 3 Forest plots of the studies estimating the HRs with corresponding $95 \%$ Cls of LMR for PFS.

Abbreviations: HR, hazard ratio; LMR, lymphocyte-to-monocyte ratio; PFS, progression-free survival; $\mathrm{Cl}$, confidence interval. 

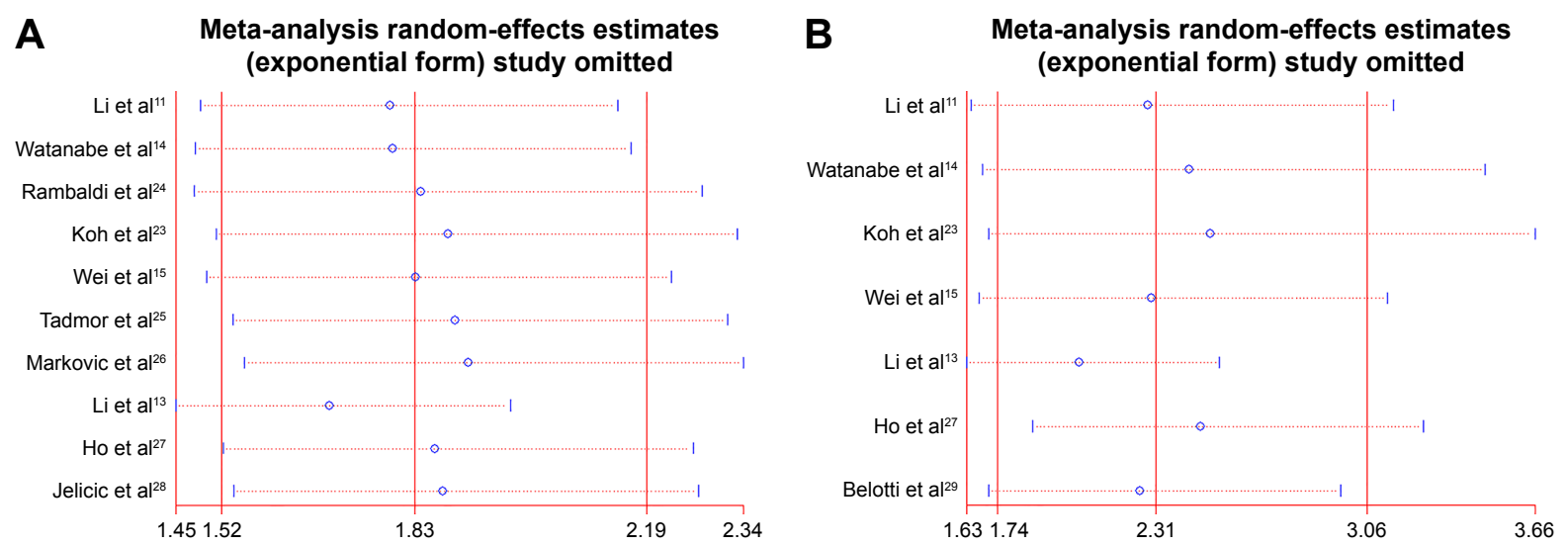

Figure 4 Sensitivity analysis of the effect of individual studies on the pooled HRs for (A) OS and (B) PFS in DLBCL.

Abbreviations: HR, hazard ratio; OS, overall survival; PFS, progression-free survival; DLBCL, diffuse large B-cell lymphoma.

\section{Heterogeneity}

In this meta-analysis, no significant heterogeneity among the studies was found for PFS $\left(P_{\mathrm{H}}<0.212\right)$ and OS $\left(P_{\mathrm{H}}=0.192\right)$, and the fixed-effect model was used to assess OS and PFS. In addition, sensitivity analysis and publication bias were also carried out to further explore the potential heterogeneity and the stability of the results among studies for OS and PFS. The pooled HRs for OS (Figure 4A) and PFS (Figure 4B) were not significantly affected by removing a single study each time. Concurrently, publication bias was not observed for OS and PFS from the Begg's funnel plot (Figure 5A and B) and Egger's test (Table 2).

\section{Discussion}

This updated meta-analysis aims to explore the associations of decreased LMR with OS and PFS in patients with DLBCL. Our results combined the survival outcomes of 4,578 patients with DLBCL extracted from eleven individual studies, suggesting that patients with DLBCL with decreased LMR had shorter OS and PFS. Subgroup analysis stratified by the country of study, cutoff value defining decreased LMR, treatment method, and sample size did not attenuate the prognostic significance of LMR in DLBCL. Despite substantial progress in the knowledge of the correlations between inflammatory response markers and survival outcomes of various cancers, the influence of inflammatory markers on tumor prognosis remains inconsistent. A previous meta-analysis that combined nine studies has shown an increased risk with low LMR from a total of 4,198 individuals. ${ }^{17}$ Our study is an updated meta-analysis covering a total of eleven published studies reporting the correlations between decreased LMR and the clinical prognosis in patients with DLBCL.

The link between inflammation and cancer has been extensively reported. Relevant mechanistic investigations also support the biologic and prognostic importance of tumor microenvironment with proinflammation in tumor
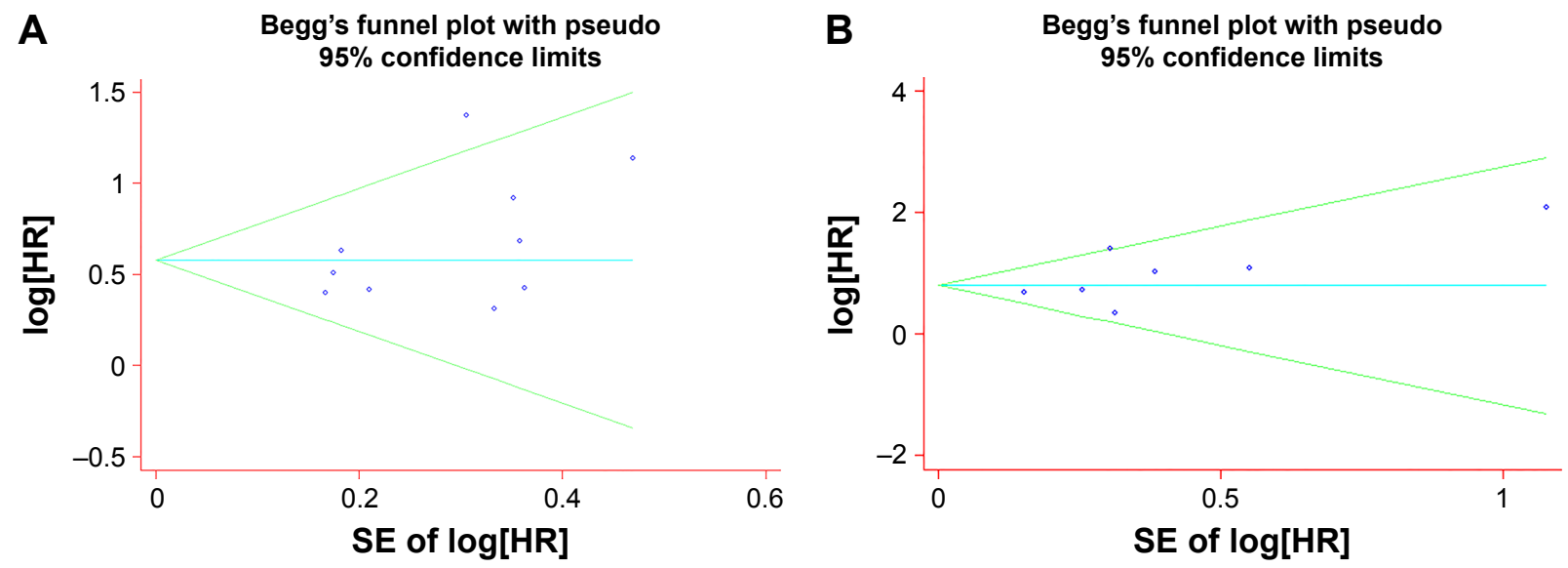

Figure 5 Begg's funnel plots for the Egger's test evaluating the publication bias for (A) OS and (B) PFS. Abbreviations: OS, overall survival; PFS, progression-free survival; HR, hazard ratio; SE, standard error. 
progression. ${ }^{5,18}$ A decreased LMR represented a decreased lymphocyte count and/or an increased monocyte count, as well as lymphopenia. Lymphocytes play an important role in tumor immunological surveillance and defense by suppressing tumor cell growth and proliferation. ${ }^{19}$ Chemokines (including CCL-2 or CCL-5 produced by tumor cells, fibroblasts, or immune cells) recruit monocytes in the tumor microenvironment. As soon as these cells encounter chemokines, tumor-associated macrophages are quickly differentiated from them and transform to a tumor-promoting M2-like macrophage polarization under certain conditions that depresses Th1-mediated inflammation through interleukin (IL)-10 and IL-1b production. Then tumor-promoting M2-like macrophage secretes different proangiogenic factors (such as vascular endothelial growth factor, IL-8, fibroblast growth factor, and matrix metalloproteinase 9) to induce angiogenesis. Therefore, LMR can present the status of pro-tumor and antitumor ability in response to inflammation, and its value combining with lymphocyte and monocyte counts index may reflect the protumor ability and antitumor capacity of the host more concisely. In addition, it is convenient and inexpensive to measure the parameter of LMR in clinical application, which makes it a fascinating marker for the prediction of DLBCL.

Recently, numerous studies have explored the prognostic significance of LMR in a variety of solid cancers, including gastric cancer, ${ }^{9}$ lung cancer, ${ }^{20}$ colorectal cancer, ${ }^{21}$ and nasopharyngeal carcinoma. ${ }^{22}$ However, few studies reported the prognostic value of LMR in DLBCL. Li et al ${ }^{11}$ suggested that LMR was an effective prognostic factor in patients with DLBCL treated with R-CHOP. Koh et $\mathrm{al}^{23}$ observed that patients with DLBCL with decreased LMR had obviously lower survival (OS and PFS) than those with elevated LMR. Wei et $\mathrm{al}^{15}$ showed that decreased LMR was not associated with the survival outcomes in patients with germinal centertype DLBCL. Lin et $\mathrm{al}^{17}$ reported that a low LMR at diagnosis had an adverse effect on outcome for patients with DLBCL according to the evidence from nine studies consisting of 4,198 subjects. Our study was an updated meta-analysis reporting the prognostic performance of LMR in patients with DLBCL, revealing that decreased LMR was used to assess clinical outcomes for patients with DLBCL, especially patients treated with R-CHOP. In addition, an advantage of LMR was that it is convenient to measure in routine testing at low cost. Therefore, LMR is a potential and promising marker for clinical application.

There are some limitations in our study. First, decreased LMR and the clinicopathologic characteristics of patients were not systematically analyzed, such as Ann-Arbor stage, bone marrow involvement, cell-of-origin subtype, and lactate dehydrogenase. Second, the number of eligible studies was relatively small. In the subgroup analyses, the studies included in each subgroup were quite few.

\section{Conclusion}

Our results suggest that decreased LMR may be an adverse prognostic factor for patients with DLBCL, which could contribute in stratification of patients and determination of individual therapeutic plans. More large-scale and welldesigned studies are warranted to better clarify the prognostic value of LMR in DLBCL.

\section{Acknowledgments}

This project was supported by grants from the National Nature Science Foundation of China (Nos 81172141 , 81200401), Nanjing Science and Technology Committee Project (No 201108025), Nanjing Medical Technology Development Project (No ZKX11025), Nanjing Health Young Talent Project, and Jiangsu Provincial Key Medical Talents to SKW; Nanjing Medical Science and Technique Development Foundation to YQP (No QRX11255) and BSH (No QRX11254); and Nanjing Medical University Science and Technique Development Foundation Project to ZLN (No 2014NJMU038) and HLS (No 2015NJMUZD049).

\section{Disclosure}

The authors report no conflicts of interest in this work.

\section{References}

1. Habermann TM. New developments in the management of diffuse large B-cell lymphoma. Hematology. 2012;17(suppl 1):S93-S97.

2. Coiffier B, Thieblemont C, Van Den Neste E, et al. Long-term outcome of patients in the LNH-98.5 trial, the first randomized study comparing rituximab-CHOP to standard CHOP chemotherapy in DLBCL patients: a study by the Groupe d'Etudes des Lymphomes de l'Adulte. Blood. 2010; 116(12):2040-2045.

3. Vegso G, Hajdu M, Sebestyen A. Lymphoproliferative disorders after solid organ transplantation-classification, incidence, risk factors, early detection and treatment options. Pathol Oncol Res. 2011;17(3): 443-454.

4. Steidl C, Lee T, Shah SP, et al. Tumor-associated macrophages and survival in classic Hodgkin's lymphoma. N Engl J Med. 2010;362(10): 875-885.

5. Hanahan D, Weinberg RA. Hallmarks of cancer: the next generation. Cell. 2011;144(5):646-674.

6. Sun H, Pan Y, He B, et al. Gene therapy for human colorectal cancer cell lines with recombinant adenovirus 5 based on loss of the insulin-like growth factor 2 imprinting. Int J Oncol. 2015;46(4):1759-1767.

7. Kim DK, Oh SY, Kwon HC, et al. Clinical significances of preoperative serum interleukin- 6 and C-reactive protein level in operable gastric cancer. BMC Cancer. 2009;9:155.

8. Kishi Y, Kopetz S, Chun YS, Palavecino M, Abdalla EK, Vauthey JN. Blood neutrophil-to-lymphocyte ratio predicts survival in patients with colorectal liver metastases treated with systemic chemotherapy. Ann Surg Oncol. 2009;16(3):614-622. 
9. Deng Q, He B, Liu X, et al. Prognostic value of pre-operative inflammatory response biomarkers in gastric cancer patients and the construction of a predictive model. J Transl Med. 2015;13:66.

10. Wada N, Zaki MA, Hori Y, et al; Osaka Lymphoma Study Group. Tumour-associated macrophages in diffuse large B-cell lymphoma: a study of the Osaka Lymphoma Study Group. Histopathology. 2012;60(2): 313-319.

11. Li ZM, Huang JJ, Xia Y, et al. Blood lymphocyte-to-monocyte ratio identifies high-risk patients in diffuse large B-cell lymphoma treated with R-CHOP. PLoS One. 2012;7(7):e41658.

12. Wilcox RA, Ristow K, Habermann TM, et al. The absolute monocyte and lymphocyte prognostic score predicts survival and identifies high-risk patients in diffuse large-B-cell lymphoma. Leukemia. 2011;25(9):1502-1509.

13. Li YL, Pan YY, Jiao Y, Ning J, Fan YG, Zhai ZM. Peripheral blood lymphocyte/monocyte ratio predicts outcome for patients with diffuse large B cell lymphoma after standard first-line regimens. Ann Hematol. 2014;93(4):617-626

14. Watanabe R, Tomita N, Itabashi M, et al. Peripheral blood absolute lymphocyte/monocyte ratio as a useful prognostic factor in diffuse large B-cell lymphoma in the rituximab era. Eur J Haematol. 2014; 92(3):204-210.

15. Wei X, Huang F, Wei Y, et al. Low lymphocyte-to-monocyte ratio predicts unfavorable prognosis in non-germinal center type diffuse large B-cell lymphoma. Leuk Res. 2014;38(6):694-698.

16. Liberati A, Altman DG, Tetzlaff J, et al. The PRISMA statement for reporting systematic reviews and meta-analyses of studies that evaluate health care interventions: explanation and elaboration. PLoS Med. 2009;6(7):e1000100

17. Lin B, Chen C, Qian Y, Feng J. Prognostic role of peripheral blood lymphocyte/monocyte ratio at diagnosis in diffuse large B-cell lymphoma: a meta-analysis. Leuk Lymphoma. 2015;56(9):2563-2568.

18. Coussens LM, Werb Z. Inflammation and cancer. Nature. 2002; 420(6917):860-867.

19. Mantovani A, Allavena P, Sica A, Balkwill F. Cancer-related inflammation. Nature. 2008;454(7203):436-444.

20. Chen YM, Lai CH, Chang HC, et al. Baseline and trend of lymphocyteto-monocyte ratio as prognostic factors in epidermal growth factor receptor mutant non-small cell lung cancer patients treated with first-line epidermal growth factor receptor tyrosine kinase inhibitors. PLoS One. 2015;10(8):e0136252.
21. Ozawa T, Ishihara S, Kawai K, et al. Impact of a lymphocyte to monocyte ratio in stage IV colorectal cancer. J Surg Res. 2015;199(2):386-392.

22. Jiang R, Cai XY, Yang ZH, et al. Elevated peripheral blood lymphocyte-to-monocyte ratio predicts a favorable prognosis in the patients with metastatic nasopharyngeal carcinoma. Chin J Cancer. 2015; 34(6):237-246.

23. Koh YW, Park CS, Yoon DH, Suh C, Huh J. Should the cut-off values of the lymphocyte to monocyte ratio for prediction of prognosis in diffuse large B-cell lymphoma be changed in elderly patients? Eur J Haematol. 2014;93(4):340-348.

24. Rambaldi A, Boschini C, Gritti G, et al. The lymphocyte to monocyte ratio improves the IPI-risk definition of diffuse large B-cell lymphoma when rituximab is added to chemotherapy. Am J Hematol. 2013;88(12): 1062-1067.

25. Tadmor T, Bari A, Sacchi S, et al. Monocyte count at diagnosis is a prognostic parameter in diffuse large B-cell lymphoma: results from a large multicenter study involving 1191 patients in the pre- and postrituximab era. Haematologica. 2014;99(1):125-130.

26. Markovic O, Popovic L, Marisavljevic D, et al. Comparison of prognostic impact of absolute lymphocyte count, absolute monocyte count, absolute lymphocyte count/absolute monocyte count prognostic score and ratio in patients with diffuse large B cell lymphoma. Eur J Intern Med. 2014;25(3):296-302.

27. Ho CL, Lu CS, Chen JH, Chen YG, Huang TC, Wu YY. Neutrophil/ lymphocyte ratio, lymphocyte/monocyte ratio, and absolute lymphocyte count/absolute monocyte count prognostic score in diffuse large B-cell lymphoma: useful prognostic tools in the rituximab era. Medicine (Baltimore). 2015;94(24):e993.

28. Jelicic J, Todorovic Balint M, Sretenovic DA, et al. Enhanced International Prognostic Index (NCCN-IPI), Charlson comorbidity index and absolute lymphocyte count as predictors for survival of elderly patients with diffuse large B cell lymphoma treated by immunochemotherapy. Neoplasma. 2015;62(6):988-995.

29. Belotti A, Doni E, Bolis S, et al. Peripheral blood lymphocyte/monocyte ratio predicts outcome in follicular lymphoma and in diffuse large B-cell lymphoma patients in the rituximab era. Clin Lymphoma Myeloma Leuk. 2015;15(4):208-213.
OncoTargets and Therapy

\section{Publish your work in this journal}

OncoTargets and Therapy is an international, peer-reviewed, open access journal focusing on the pathological basis of all cancers, potential targets for therapy and treatment protocols employed to improve the management of cancer patients. The journal also focuses on the impact of management programs and new therapeutic agents and protocols on

\section{Dovepress}

patient perspectives such as quality of life, adherence and satisfaction. The manuscript management system is completely online and includes a very quick and fair peer-review system, which is all easy to use. Visit http://www.dovepress.com/testimonials.php to read real quotes from published authors. 